\title{
Design Simulation and Preparation of White OLED Microdisplay Based on Microcavity Structure Optimization
}

\author{
Liangfei Duan (D), Guanghua Wang, Yu Duan, Denglin Lei, Fuli Qian, and Qiming Yang \\ Yunnan OLiGHTEK Opto-Electronic Technology Co. Ltd., Kunming 650214, China \\ Correspondence should be addressed to Liangfei Duan; liangfeiduan1012@163.com
}

Received 21 February 2021; Revised 8 April 2021; Accepted 28 April 2021; Published 10 May 2021

Academic Editor: Alessandra Durazzo

Copyright (c) 2021 Liangfei Duan et al. This is an open access article distributed under the Creative Commons Attribution License, which permits unrestricted use, distribution, and reproduction in any medium, provided the original work is properly cited.

\begin{abstract}
White-light OLED devices play an important application in information display fields. Optical interference of the microcavity structure has an important effect on device performances. According to the design of the band structure, $\mathrm{ITO}_{\mathrm{MoO}} \mathrm{M}$ composite films were used as the anode, and $\mathrm{Mg}$ : $\mathrm{Ag}(1 \%)$ composite films were prepared by coevaporation as the translucent cathode; $\mathrm{CuPc}$ was used as the hole injection layer and anode passivation layer, NPB as the hole transmission layer and yellow light main material, rubrene as yellow dopant material, $\mathrm{ADN}$ as blue light main material, DSA-Ph as blue dopant material, and TPBi and Alq3 as the electron transport layers. We realized the change of the microcavity structure by adjusting the thickness of each organic functional layer film and simulated and calculated the optimized thickness of each organic film layer and influence on OLED device performances using the SimOLED software system. The optimized OLED microdisplay structure is $\mathrm{Si}_{(\mathrm{CMOS})} / \mathrm{ITO}(35 \mathrm{~nm}) / \mathrm{MoO}_{3}$ $(2 \mathrm{~nm}) / \mathrm{CuPc}(5 \mathrm{~nm}) / 2$-TNATA $(20 \mathrm{~nm}) / \mathrm{NPB}(10 \mathrm{~nm}) / \mathrm{NPB}:$ rubrene $(1.5 \%) \mathrm{ADN}: \mathrm{DSA}-\mathrm{Ph}(5 \%)(25 \mathrm{~nm}) / \mathrm{TPBi}(15 \mathrm{~nm}) / \mathrm{Alq} 3$ $(1.2 \mathrm{~nm}) / \mathrm{Mg}(13 \mathrm{~nm}): \mathrm{Ag}(1 \%)$. The optimized OLED microdisplay was prepared by the vacuum coating system, and the photoelectric performances of the OLED device were characterized by a spectral testing system consisting of the Photo Research PR655 spectrometer and Keithley 2400 program-controlled power supply. The effect of the microcavity structure on OLED device performances was studied. The results show that the variation of the film thickness of each organic functional layer has an important effect on the performances of OLED microdisplay, such as brightness and color coordinate, and the OLED microdisplay reaches a higher brightness of $3342 \mathrm{~cd} / \mathrm{m}^{2}$ under the normal working voltage at $5.0 \mathrm{~V}$ after the structure is optimized, with CIE coordinate $(0.28,0.37)$, which is closer to the energy point of standard white light.
\end{abstract}

\section{Introduction}

Organic light-emitting diodes (OLEDs) with the features such as lower drive voltage, higher brightness, wider viewing angle, and better temperature adaptability $[1,2]$ are recognized as the next-generation display technology after CRT and LCD. OLED microdisplays are integrated of silicon-based CMOS technologies and OLED technologies, which perform important application prospects in the fields of military, aerospace, and consumer electronics (AR and VR) $[3,4]$.

White OLED can be realized as full-color display through the color filter layer (RGB) and solid-state lighting source, which has attracted wide attention [5-7]. Organic materials that direct emitting white light are rare and have poor performances in the OLED material system [8]. We usually adopt the method of multisource coevaporation and introduce a certain proportion of doped materials into the main material to achieve the white OLED device [9]. Highefficiency white organic light-emitting diodes with high color stability together with reduced efficiency roll off by wisely utilizing combinations of exciton-managed orangephosphorescence/sky blue-fluorescence with the peak current efficiencies of $42.5 \mathrm{~cd} / \mathrm{A}$ [10]. White organic lightemitting devices were fabricated using an ultrathin layer, with the maximum current efficiency of $8.69 \mathrm{~cd} / \mathrm{A}$ at $7 \mathrm{~V}$ [11]. Nondoped white organic light-emitting devices with a quadruple-quantum-well structure were fabricated, and an alternate layer of ultrathin blue and yellow iridium complexes was employed as the potential well layer, with the luminous efficiency of $10.2 \mathrm{~cd} / \mathrm{A}$ [12]. An ultrahigh color rendering index (CRI) white organic light-emitting device was achieved by using a deep red phosphorescent dye; the 
WOLED exhibits high luminance of $2529 \mathrm{~cd} / \mathrm{m}^{2}$ at $5 \mathrm{~V}$ [13]. White organic light-emitting devices with fluorescent donor-acceptor-substituted spirobifluorene compounds have been fabricated, with the maximum current efficiency of $5.9 \mathrm{~cd} / \mathrm{A}$ [14]. The multilayer structure of phosphorescent organic white light-emitting devices was fabricated, and the spectral stability was enhanced by adding a hole-blocking layer in the light-emitting layer, and the current efficiency is $24.7 \mathrm{~cd} / \mathrm{A}$ [15]. Vacuum-evaporated Ag-island nanostructures were incorporated into the electron-transporting layer (ETL), and their effects on the performance of nondoped blue phosphorescent organic light-emitting devices (PhOLEDs) using an ultrathin phosphorescent dye as the emitting layer (EML) were investigated systematically; the current efficiency of the optimized device was enhanced by $54 \%$ [16]. Highly efficient nondoped tandem phosphorescent organic light-emitting devices (PhOLEDs) have been demonstrated by employing $\mathrm{LiF} / \mathrm{AL} / 1,4,5,8,9,11$-hexaazatriphenylene hexacarbonitrile (HAT-CN) as a charge generation unit (CGU) and ultrathin phosphorescent dyes as emitting layers (EMLs), with the maximum current efficiency of $94.9 \mathrm{~cd} / \mathrm{A}$ and maximum external quantum efficiency of $31.6 \%$ [17]. Solution-processed small-molecule white organic light-emitting diodes were fabricated with a cohost of hole transporter (TCTA) and electron transporter (SPPO13), with the maximum efficiencies of $40.9 \mathrm{~cd} / \mathrm{A}$ at the practical luminance of $1000 \mathrm{~cd} / \mathrm{m}^{2}$ [18]. The acquisition of white OLED devices was mostly achieved by doping and mixing a variety of colors. The blue light and yellow light are mixed and complementary, or red, green, and blue colors are mixed and complementary to achieve white light $[19,20]$. Therefore, the structure of the white OLED microdisplay is composed of a variety of organic functional films. The microcavity produced by the semitransparent cathode and the highly reflective anode has a great influence on the performances of the OLED microdisplay. The structures and performances of each film layer of OLED microdisplay are different, and the thickness and matching of each organic functional film will directly affect the length of the microcavity and the position of the luminescent body in the microcavity, which will lead to the shift of the brightness and color coordinates. In order to improve the performances of white-light OLED microdisplay, we designed $\mathrm{Si}_{(\mathrm{CMOS})} / \mathrm{ITO} \quad(35 \mathrm{~nm}) / \mathrm{MoO}_{3} \quad(2 \mathrm{~nm}) / \mathrm{CuPc}$ $(5 \mathrm{~nm}) / 2$-TNATA $\quad(20 \mathrm{~nm}) / \mathrm{NPB} \quad(10 \mathrm{~nm}) / \mathrm{NPB}$ : rubrene $(1.5 \%)$ ADN : DSA-Ph $\quad(5 \%) \quad(25 \mathrm{~nm}) / \mathrm{TPBi} \quad(15 \mathrm{~nm}) / \mathrm{Alq} 3$ $(1.2 \mathrm{~nm}) / \mathrm{Mg}(13 \mathrm{~nm}): \mathrm{Ag}(1 \%)$ as the main light-emitting structure of OLED microdisplays. The SimOLED software system was used to simulate the matching of organic functional films and OLED device structure optimization to study the effect of the microcavity structure on the performances of OLED microdisplays. According to the simulation results, the high-efficiency white OLED microdisplays were prepared by coevaporation doped with blue and yellow doped materials, respectively.

\section{Experimental}

2.1. Design and Simulation of OLED Microdisplay. The structure of OLED microdisplay was designed according to the material band matching and device structure of OLED microdisplay. The theme structure is $\mathrm{Si}_{(\mathrm{CMOS})} / \mathrm{ITO} / \mathrm{MoO}_{3} /$ $\mathrm{CuPc} / 2$-TNATA/NPB/NPB : rubrene (1.5\%)ADN : DSA-Ph (5\%)/TPBi/Alq3/Mg:Ag (1\%). The SimOLED software system was used to simulate and optimize the effects of the thickness of each organic functional film layer, microcavity structure, and light-emitting point position on the performances of OLED microdisplay.

2.2. Fabrication of OLED Microdisplay. White OLED microdisplays were prepared by mixing yellow with blue light, $\mathrm{CuPc}$ as the hole injection layer and anode passivation layer, NPB as hole transmission material, and rubrene as yellow light dopant material with a peak spectrum at $560 \mathrm{~nm}$; NPB was used as yellow light main material. The yellow light-emitting layer was prepared by coevaporation of NPB : rubrene (1.5\%). DSA-Ph was used as blue light dopant material with a peak spectrum at $470 \mathrm{~nm}$ and $500 \mathrm{~nm}$; ADN was used as the blue light main material. The blue lightemitting layer was prepared by coevaporation of $\mathrm{ADN}$ : DSA-Ph (5\%). TPBi and Alq3 were used as electron transport materials, and $\mathrm{Mg}: \mathrm{Ag}(1 \%)$ composite films were prepared by coevaporation as the translucent cathode. The WOLED microdisplay was prepared by the vacuum coating system.

2.3. Performance Characterization. The thickness and optical parameters (refractive index $n$ and extinction coefficient $k$ ) of the organic films were measured by SCI FilmTek SE ellipsometry, and the luminescence spectrum of the emitting layer film was measured by using the F55 spectrofluorometer. The emission spectrum, voltage, brightness, color coordinates, and other parameters of green OLED devices were tested using the OLED test system, which was composed of the Photo Research PR655 spectrometer and Keithley 2400 programmable power supply for synchronous measurement.

\section{Results and Discussion}

OLED microdisplay is a top-emitting OLED device. The anode needs to have the characteristics of high work function, high reflectivity, and corrosion resistance, and the cathode needs to have the characteristics of low work function and good transmittance. According to the characteristics of materials, device structure, and energy, Si (CMOS) was used as the substrate, and $\mathrm{ITO} / \mathrm{MoO}_{3}$ composite films were used as the anode of OLED microdisplay because of $\mathrm{MoO}_{3}$ with high power function and corrosion resistance; it can also block the damage of OLED devices due to the diffusion of In in ITO. CuPc was used as the hole injection layer and anode passivation layer, NPB as hole transmission material and yellow light main material, and rubrene as yellow dopant material. DSA-Ph was used as blue light dopant material, and ADN was used as the blue light main material. TPBi and Alq3 were used as electron transport materials, and $\mathrm{Mg}: \mathrm{Ag}(1 \%)$ composite films were used as the translucent cathode. The structure is ITO/ $\mathrm{MoO}_{3} /$ $\mathrm{CuPc} / 2$-TNATA/NPB/NPB : rubrene (1.5\%) ADN : DSA-Ph 


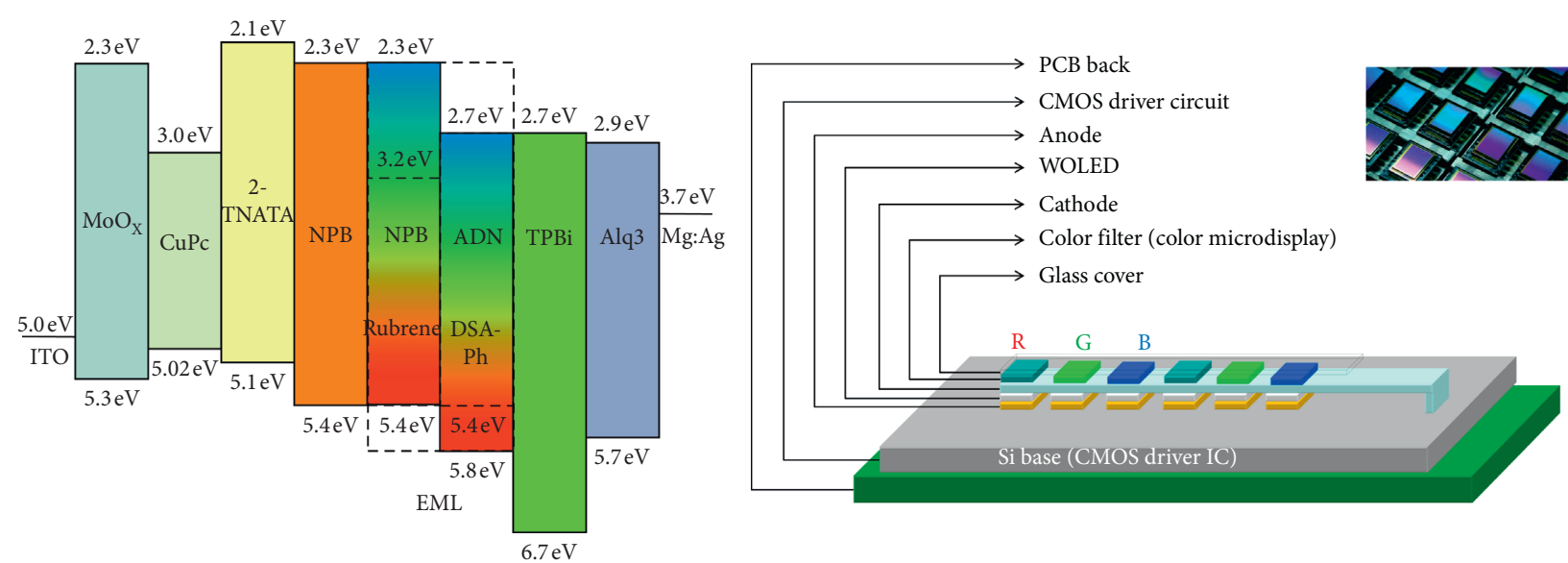

Figure 1: Materials and structure of OLED microdisplay.

(5\%)/TPBi/Alq3/Mg:Ag (1\%). The device structure, material, and energy-matching diagram have been shown in Figure 1.

The database was established based on the refractive index $(n)$, extinction coefficient $(k)$, and luminescence spectrum (PL) of organic material functional films, and simulation models were built according to the structure of OLED microdisplay. White OLED microdisplays were prepared by mixing yellow with blue light. The SimOLED software system was used to simulate and optimize the effects of the thickness of each organic functional film layer, microcavity structure, and light-emitting point position on the performances of OLED microdisplay.

As illustrated in Figure 2, the CuPc material film of hole injection layer thickness increased from $1 \mathrm{~nm}$ to $10 \mathrm{~nm}$, the brightness of OLED microdisplay increased gradually and tended to be flat again, and the CIE coordinates gradually drifted towards the white light equal-energy point CIE $(0.33$, 0.33). The 2-TNATA material film of hole transport layer thickness increased from $10 \mathrm{~nm}$ to $30 \mathrm{~nm}$, the brightness of OLED microdisplay increased gradually and then decreased, and the CIE coordinates gradually drifted towards the white light equal-energy point. The NPB material film of hole transport layer thickness increased from $6 \mathrm{~nm}$ to $14 \mathrm{~nm}$, the brightness of OLED microdisplay increased linearly, and the CIE coordinates had less drift. NPB: rubrene (1.5\%) and $\mathrm{ADN}$ : DSA-Ph (5\%) materials' film of emitting layer thickness increased from $8 \mathrm{~nm}$ to $20 \mathrm{~nm}$, the brightness of OLED microdisplay increased linearly, and the CIE coordinates had less drift. TPBi material film of electron transport layer thickness increased from $15 \mathrm{~nm}$ to $30 \mathrm{~nm}$, the brightness of OLED microdisplay decreased linearly, and the CIE coordinates gradually drifted towards the white light equal-energy point. Alq3 material film of electron injection layer thickness increased from $1 \mathrm{~nm}$ to $4 \mathrm{~nm}$, the brightness of OLED microdisplay decreased linearly, and the CIE coordinates gradually drifted towards the white light equalenergy point.

As illustrated in Figure 3, $\mathrm{Si}$ (CMOS) was used as the substrate, and ITO/MoO 3 composite films were used as the anode of OLED microdisplay. The anode has high reflectivity. $\mathrm{Mg}: \mathrm{Ag}(1 \%)$ composite films were used as the translucent cathode, and the cathode also has some reflections. The microcavity is formed between the highly reflective anode and the semitransparent cathode.

Microcavity resonance can cause optical interference inside OLED devices, which affects OLED device performances. The thickness, refractive index $(n)$, and extinction coefficient $(k)$ of each organic functional layer film between parallel electrodes will directly affect the length of the microcavity and the position of the luminescent point in the microcavity, which affect the microcavity structure and OLED device performances [21, 22]. Therefore, the microcavity length and the position of the luminescent body in the microcavity can be controlled by changing the thickness of each organic functional layer film and the optimal matching between them, and then the brightness and CIE coordinates of OLED microdisplay can be controlled.

The optimized structure of OLED microdisplay was obtained according to the simulation calculation results by SimOLED. The structure is $\mathrm{Si}_{(\mathrm{CMOS})} / \mathrm{ITO}(35 \mathrm{~nm}) / \mathrm{MoO}_{3}$ $(2 \mathrm{~nm}) / \mathrm{CuPc}(5 \mathrm{~nm}) / 2-\mathrm{TNATA} \quad(20 \mathrm{~nm}) / \mathrm{NPB} \quad(10 \mathrm{~nm}) /$ NPB : rubrene (3.5\%) ADN (15 nm):DSA-Ph (35\%) (25 nm)/TPBi (15 nm)/Alq3 (1.2 nm)/Mg (13 nm): Ag (1\%). The structures and performances of the optimized and matched OLED microdisplay were simulated and calculated by SimOLED software, and the results are shown in Figure 4.

As illustrated in Figure 4, the brightness of the OLED microdisplay has improved greatly, and the CIE coordinates drift further towards the white light energy point after the films' thickness and optical matching of each organic functional layer. The optimized OLED device structure was prepared by vacuum coating technology, and the performances of the OLED device before and after optimization were analyzed and compared.

Figure 5 illustrates the J-V characteristic curve and L-V curve of white OLED microdisplay before and after optimization.

WOLED1 (optimized OLED microdisplay structure): $\mathrm{Si}_{\text {(CMOS) }} / \mathrm{ITO} \quad(35 \mathrm{~nm}) / \mathrm{MoO}_{3} \quad(2 \mathrm{~nm}) / \mathrm{CuPc} \quad(5 \mathrm{~nm}) / 2-$ TNATA $(20 \mathrm{~nm}) / \mathrm{NPB}(10 \mathrm{~nm}) / \mathrm{NPB}$ : rubrene $(3.5 \%) \mathrm{ADN}$ : 

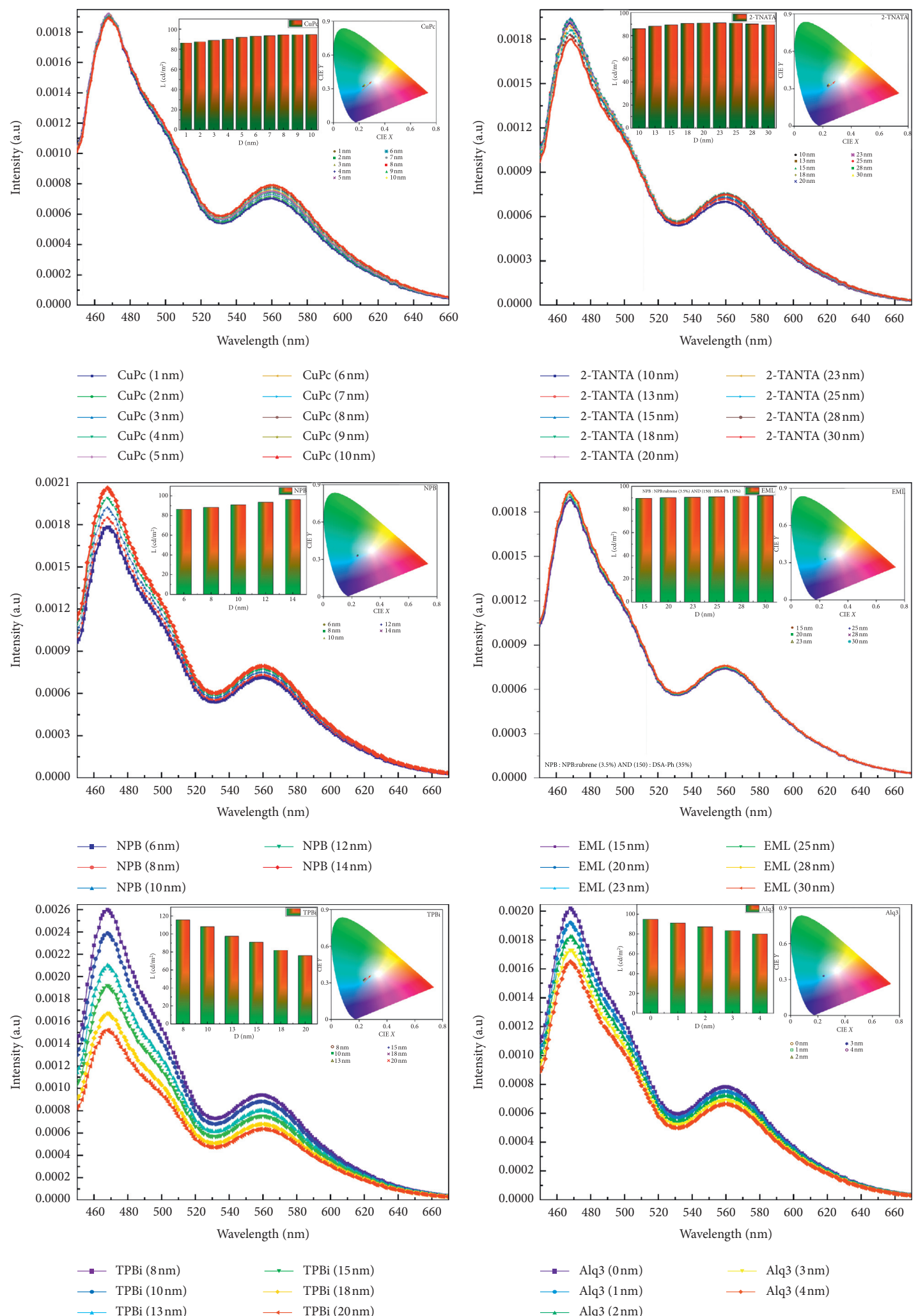

FIgURE 2: The influence of the thickness of organic films on the performances of OLED microdisplay. 


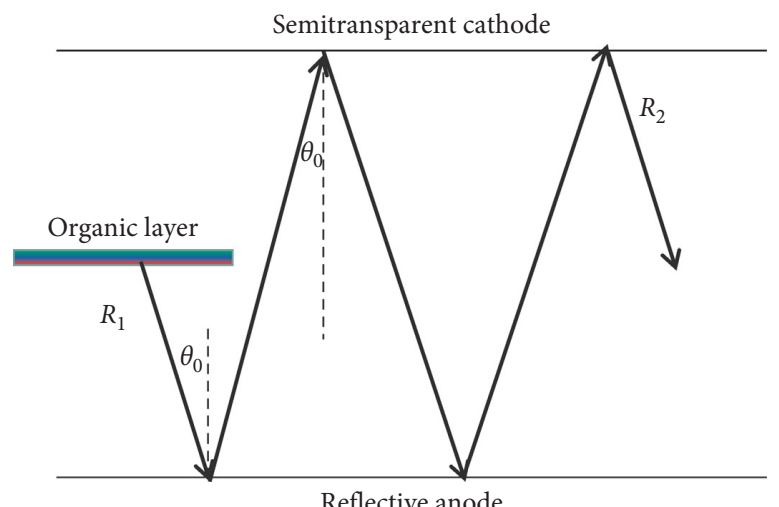

Figure 3: Microcavity structure of OLED microdisplay.
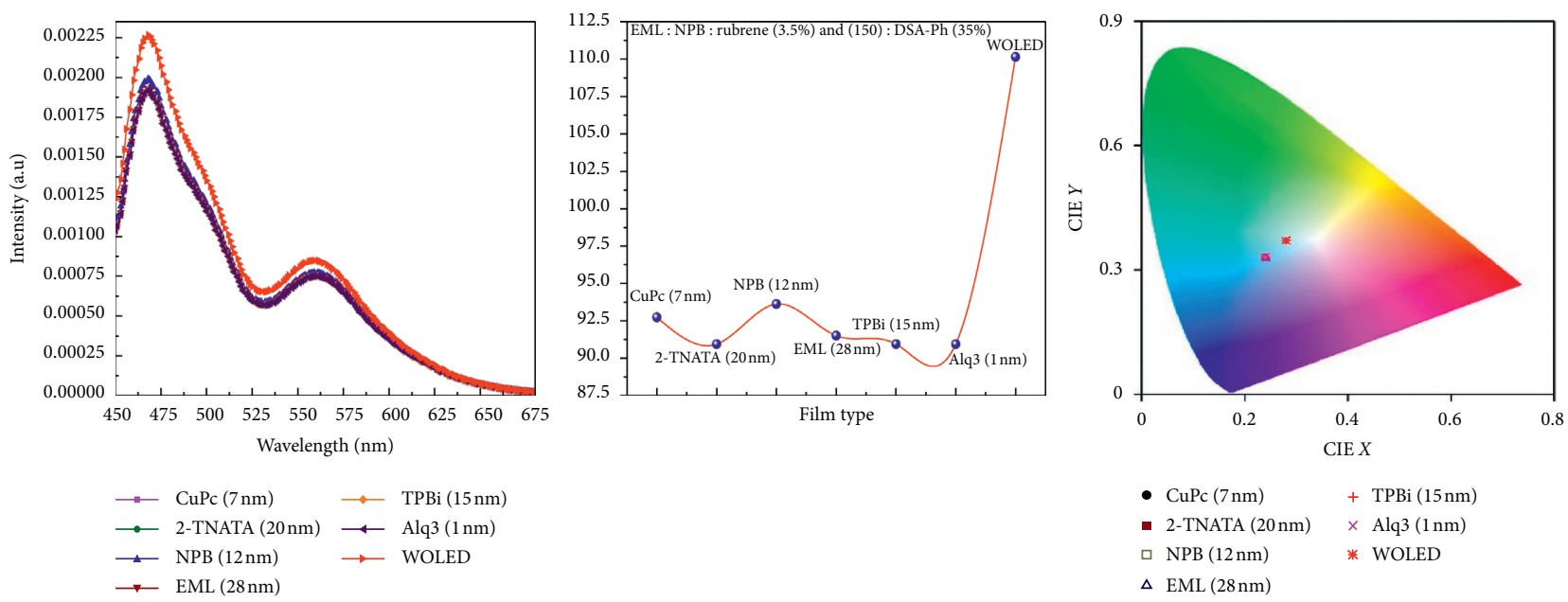

FIGURE 4: Optimized OLED microdisplay performances.

DSA-Ph (35\%) $(25 \mathrm{~nm}) / \mathrm{TPBi}(15 \mathrm{~nm}) / \mathrm{Alq} 3(1.2 \mathrm{~nm}) / \mathrm{Mg}$ $(13 \mathrm{~nm}): \mathrm{Ag}(1 \%)$.

WOLED2 (previous OLED microdisplay structure): $\mathrm{Si}_{\text {(CMOS })} / \mathrm{ITO} \quad(35 \mathrm{~nm}) / \mathrm{MoO}_{3} \quad(2 \mathrm{~nm}) / \mathrm{CuPc} \quad(8 \mathrm{~nm}) / 2-$ TNATA $(18 \mathrm{~nm}) / \mathrm{NPB}(12 \mathrm{~nm}) / \mathrm{NPB}$ : rubrene $(3.5 \%) \mathrm{ADN}$ : DSA-Ph $\quad(35 \%) \quad(28 \mathrm{~nm}) / \mathrm{TPBi} \quad(10 \mathrm{~nm}) / \mathrm{Alq} 3 \quad(2 \mathrm{~nm}) / \mathrm{Mg}$ $(13 \mathrm{~nm}): \mathrm{Ag}(1 \%)$.

White OLED microdisplay has a rectifier effect, and the current varies little with the voltage in low voltage; when a certain voltage is exceeded, the current density increases sharply with the voltage. The optimized OLED microdisplay has a lower starting voltage than the original OLED microdisplay and higher current density and brightness under the same voltage. The brightness reached $3342 \mathrm{~cd} / \mathrm{m}^{2}$ at a normal working voltage of $5 \mathrm{~V}$, and the current efficiency was $9.28 \mathrm{~cd} / \mathrm{A}$ after the structure of white OLED microdisplay was optimized.
As illustrated in Figure 6 and Table 1, the spectrum of white OLED microdisplays was measured under the current density of $20 \mathrm{~mA} / \mathrm{cm}^{2}$, and the analysis was performed by Gaussian fits. The white OLED devices obtained two blue light peaks near $470 \mathrm{~nm}$ and $500 \mathrm{~nm}$ and one yellow light peak near $560 \mathrm{~nm}$. The optimized OLED microdisplay blue shift of the blue emission peak at $470 \mathrm{~nm}$ is reduced, peak intensity and area are increased, and the FWHM width is gradually reduced. The blue shift offset of the blue peak $(500 \mathrm{~nm})$ relatively increased, while the FWHM width and peak area relatively decreased. The red shift offset, FWHM width, and peak area of the yellow peak $(560 \mathrm{~nm})$ relatively increased. The CIE coordinates $(0.28,0.37)$ drift further towards the white light energy point. The performance of the optimized OLED microdisplay accords with the simulation results.

As illustrated in Figure 7, white OLED microdisplays were prepared by vacuum evaporation technology. White 


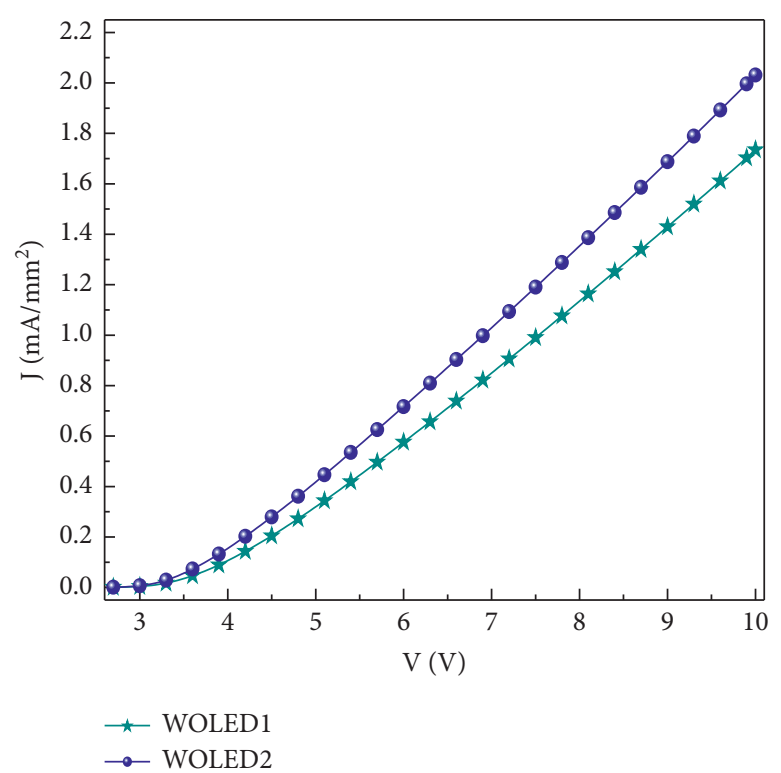

(a)

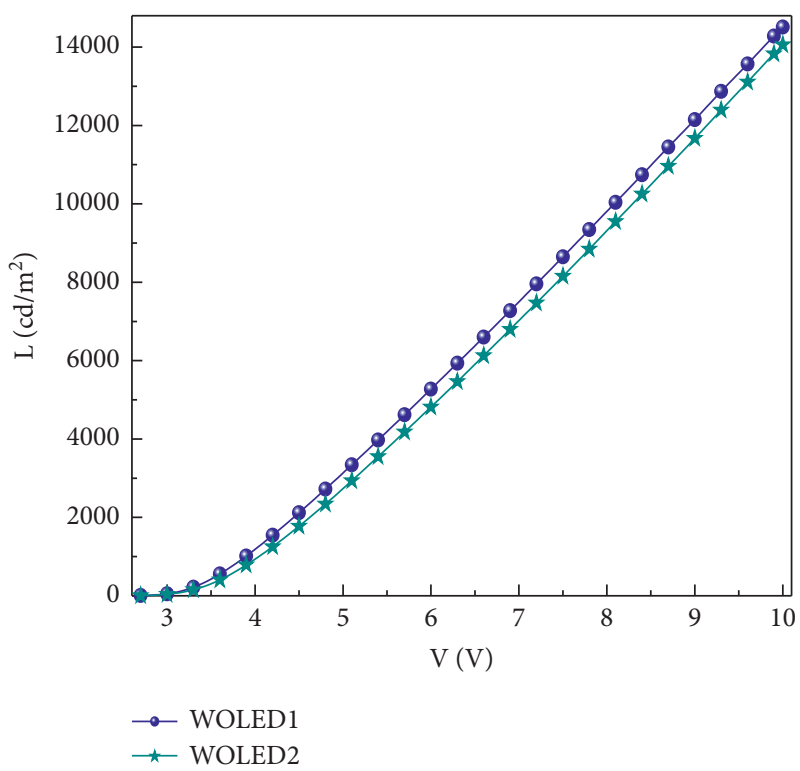

(b)

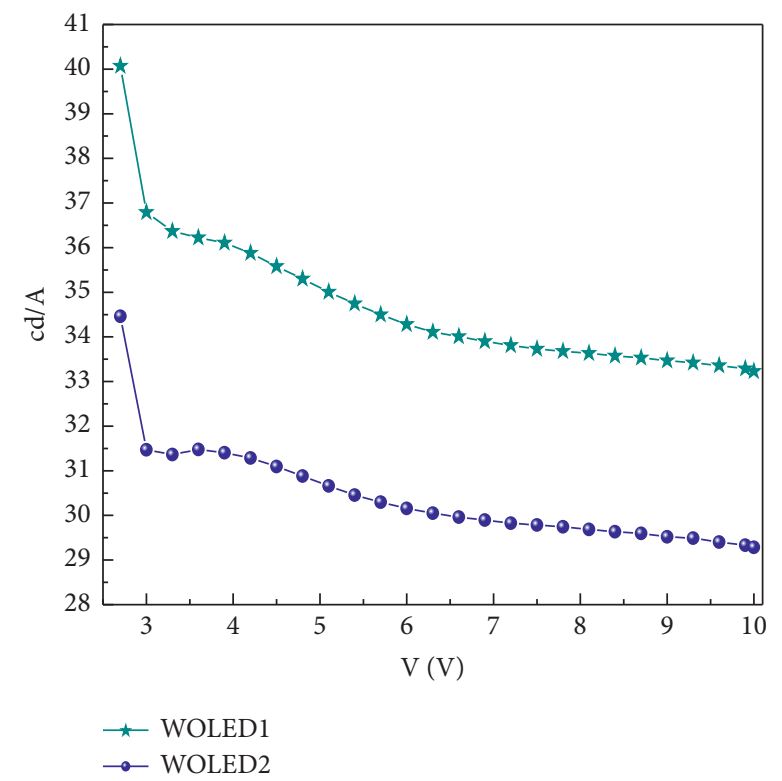

(c)

FIGURE 5: (a) J-V characteristic curves of white OLED, (b) L-V characteristic curves of white OLED, and (c) light-emitting efficiency of white OLED.

OLED devices consist of PCB backplane, IC driving circuit, anode, OLED organic thin film layers, and cathode. Each pixel of the white OLED device is composed of red, green, and blue subpixels. The size of a single pixel is $15 \times 15 \mu \mathrm{m}$, the duty cycle is $75 \%$, and the display area is $12.06 \times 9.06 \mathrm{~mm}$. The brightness and CIE coordinates are changed because of the changes of the microcavity structure and the position of the emitting body in the microcavity by different OLED microdisplays [23]. The OLED microdisplay with higher efficiency and higher brightness has been obtained by combining with simulation calculation and experimental research. 


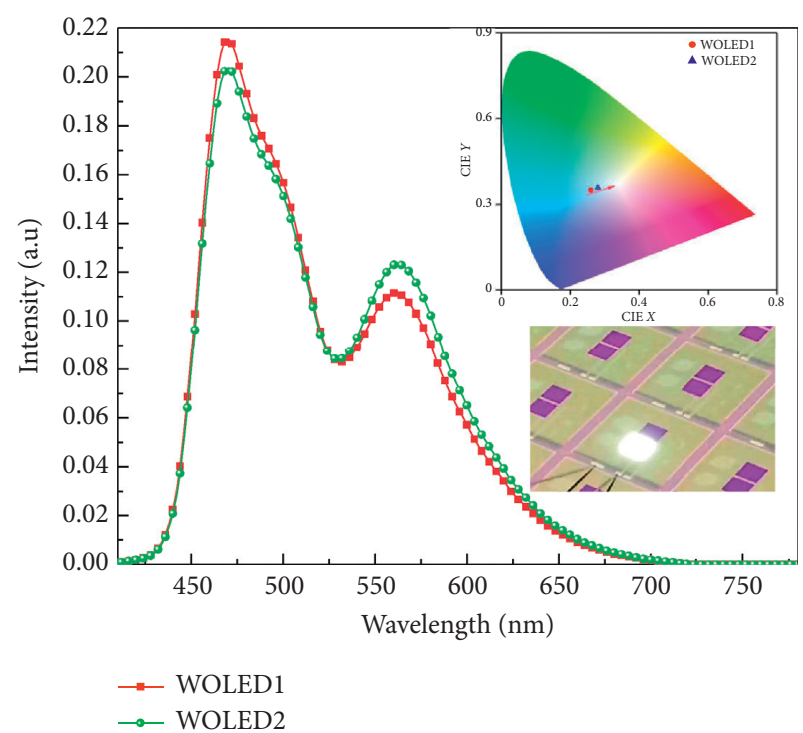

(a)

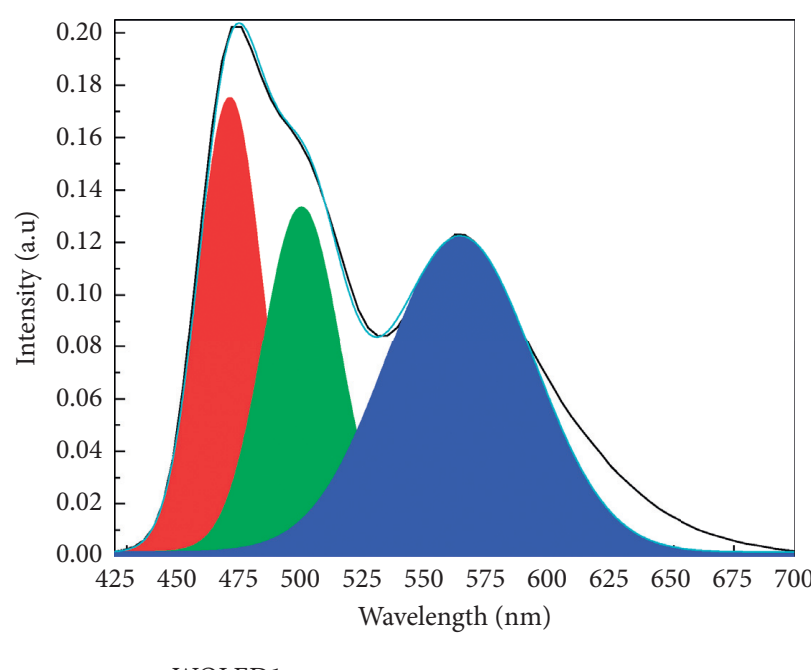

(b)

FIgURE 6: (a) Luminescence spectra of WOLED. (b) Peak fitting of WOLED luminescence spectra.

TABLE 1: Spectral parameters of white OLED devices.

\begin{tabular}{|c|c|c|c|c|c|c|c|c|c|}
\hline \multirow{4}{*}{ Devices } & \multicolumn{9}{|c|}{ Peak fit } \\
\hline & \multirow{2}{*}{\multicolumn{3}{|c|}{$\begin{array}{l}\text { Peak } 1 \\
470 \mathrm{~nm}\end{array}$}} & \multirow{2}{*}{\multicolumn{3}{|c|}{$\begin{array}{l}\text { Peak } 2 \\
500 \mathrm{~nm}\end{array}$}} & \multirow{2}{*}{\multicolumn{3}{|c|}{$\begin{array}{l}\text { Peak } 3 \\
560 \mathrm{~nm}\end{array}$}} \\
\hline & & & & & & & & & \\
\hline & $\lambda(\mathrm{nm})$ & FWHM (nm) & A & $\lambda(\mathrm{nm})$ & FWHM (nm) & A & $\lambda(\mathrm{nm})$ & FWHM (nm) & A \\
\hline WOLED (1) & 467.91 & 30.43 & 6.35 & 497.96 & 36.99 & 5.53 & 561.02 & 71.30 & 7.96 \\
\hline WOLED (2) & 467.94 & 30.04 & 5.36 & 496.97 & 36.86 & 5.10 & 562.19 & 72.07 & 9.53 \\
\hline
\end{tabular}

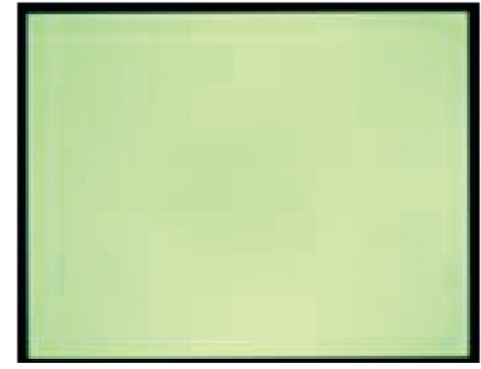

WOLED 1

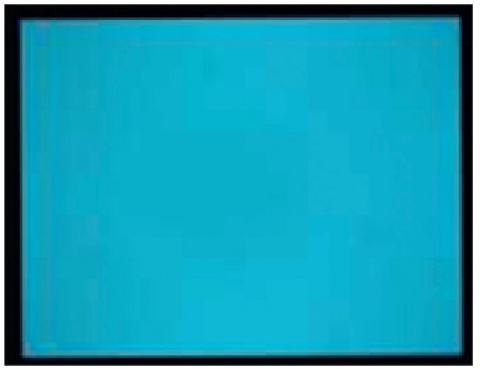

WOLED2

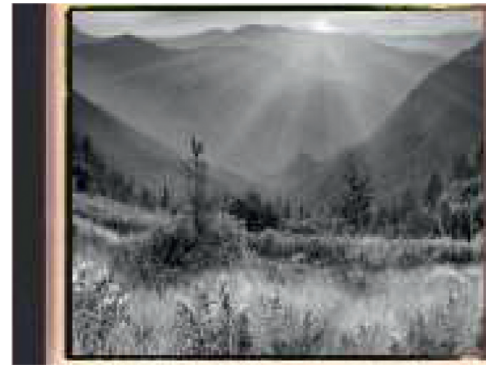

Microdisplay

Figure 7: White OLED microdisplay cell.

\section{Conclusions}

In conclusion, high-efficiency and high-brightness white OLED microdisplay has been obtained by combining with simulation calculation and experimental research. By changing the thickness of each organic functional layer film, the microcavity structure and the position of the luminescence body in the microcavity were adjusted. The SimOLED software system was used to optimize the thickness of each organic functional layer film, and the structure of the device was optimized according to the energy, thickness, and optical matching. The OLED microdisplay reaches a brightness of $3342 \mathrm{~cd} / \mathrm{m}^{2}$ under the normal working voltage at $5.0 \mathrm{~V}$ after the structure is optimized, with a current efficiency of $9.28 \mathrm{~cd} / \mathrm{A}$ and CIE coordinate $(0.28,0.37)$, which is closer to the energy point of standard white light. The results in this work could also give valuable clues on the OLED microcavity structures, high-efficiency and high-brightness OLED microdisplay, and next-generation display technologies with solution processing. 


\section{Data Availability}

The data used to support the findings of this study are included within the article.

\section{Conflicts of Interest}

The authors declare that they have no conflicts of interest.

\section{Acknowledgments}

The authors gratefully acknowledge the financial support from Yunnan Fundamental Research Projects (2019-1-C25318000002171) and Postgraduate Research Innovation Project of Yunnan University (2020174).

\section{References}

[1] G. Xie, Z. Zhang, Q. Xue et al., "Highly efficient top-emitting white organic light-emitting diodes with improved contrast and reduced angular dependence for active matrix displays," Organic Electronics, vol. 11, no. 12, pp. 2055-2059, 2010.

[2] X.-Y. Jiang, Z.-L. Zhang, J. Cao, M. A. Khan, u.-H. Khizar, and W.-Q. Zhu, "White OLED with high stability and low driving voltage based on a novel buffer layer MoOx," Journal of Physics D: Applied Physics, vol. 40, no. 18, pp. 5553-5557, 2007.

[3] B. Richter, U. Vogel, and P. Wartenberg, "OLED-on-CMOS based bidirectional microdisplay for near-to-eye and sensor applications," in Proceedings of Semiconductor Conference Dresden IEEE, pp. 1-3, Dresden, Germany, September 2011.

[4] G. B. Levy, W. Evans, J. Ebner et al., “An $852 \times 600$ pixel OLED-on-silicon color microdisplay using CMOS subthreshold-voltage-scaling current drivers," IEEE Journal of Solid-State Circuits, vol. 37, no. 12, pp. 1879-1889, 2002.

[5] C.-H. Chang, K.-C. Tien, C.-C. Chen et al., "Efficient phosphorescent white OLEDs with high color rendering capability," Organic Electronics, vol. 11, no. 3, pp. 412-418, 2010.

[6] J. Zhou, N. Ai, L. Wang et al., "Roughening the white OLED substrate's surface through sandblasting to improve the external quantum efficiency," Organic Electronics, vol. 12, no. 4, pp. 648-653, 2011.

[7] C.-H. Chang, C.-L. Ho, Y.-S. Chang et al., "Blue-emitting Ir (iii) phosphors with 2-pyridyl triazolate chromophores and fabrication of sky blue- and white-emitting OLEDs," Journal of Materials Chemistry C, vol. 1, no. 15, pp. 2639-2647, 2013.

[8] Y. Zhao, L. Zhu, J. Chen, and D. Ma, "Improving color stability of blue/orange complementary white OLEDs by using single-host double-emissive layer structure: comprehensive experimental investigation into the device working mechanism," Organic Electronics, vol. 13, no. 8, pp. 1340-1348, 2012.

[9] B. W. D'Andrade, R. J. Holmes, and S. R. Forrest, "Efficient organic electrophosphorescent white-light-emitting device with a triple doped emissive layer," Advanced Materials, vol. 16, no. 7, pp. 624-628, 2010.

[10] Q. Wang, C.-L. Ho, Y. Zhao, D. Ma, W.-Y. Wong, and L. Wang, "Reduced efficiency roll-off in highly efficient and color-stable hybrid WOLEDs: the influence of triplet transfer and charge-transport behavior on enhancing device performance," Organic Electronics, vol. 11, no. 2, pp. 238-246, 2010.

[11] X. Qin, S. Zhang, G. Xie et al., "Efficient fluorescent white organic light-emitting devices based on a ultrathin 5, 6, 11, 12- tetraphenylnaphthacene layer," Solid-State Electronics, vol. 57, no. 1, pp. 35-38, 2011.

[12] J. Zhao, J. Yu, L. Zhang, and J. Wang, "Non-doped phosphorescent white organic light-emitting devices with a quadruple-quantum-well structure," Physica B: Condensed Matter, vol. 407, no. 14, pp. 2753-2757, 2012.

[13] Li Yang, W. Zhang, L. Zhang et al., "Ultra-high general and special color rendering index white organic light-emitting device based on a deep red phosphorescent dye," Organic Electronics, vol. 14, no. 12, pp. 3201-3205, 2013.

[14] T. Zhang, T. Li, W. Hu, W. Wang, L. Zhang, and W. Xie, "Efficient fluorescent white organic light-emitting devices with a reduced efficiency roll-off based on a blue ambipolar fluorescent emitter," Current Applied Physics, vol. 14, no. 5, pp. 680-684, 2014.

[15] X. H. Liu, J. J. Yang, and J. S. Yu, "Enhancement of woleds spectral stability by adding hole-blocking interlayer," Chinese Journal of Luminescence, vol. 36, no. 10, pp. 1145-1149, 2015.

[16] X. Zhang, J. Zhang, J. Wang et al., "Efficient non-doped blue phosphorescent organic light-emitting devices by incorporating Ag-island nanostructures," Organic Electronics, vol. 58, pp. 25-32, 2018.

[17] X. Zhang, M. Zhang, M. Liu et al., "Highly efficient tandem organic light-emitting devices adopting a nondoped chargegeneration unit and ultrathin emitting layers," Organic Electronics, vol. 53, pp. 353-360, 2018.

[18] X. Du, X. Yang, J. Zhao, H. Lin, C. Zheng, and S. Tao, "Highly efficient solution-processed small-molecule white organic light-emitting diodes," Organic Electronics, vol. 38, no. 11, pp. 344-349, 2016.

[19] C.-H. Chang, H.-C. Cheng, Y.-J. Lu et al., "Enhancing color gamut of white OLED displays by using microcavity green pixels," Organic Electronics, vol. 11, no. 2, pp. 247-254, 2010.

[20] J.-H. Jou, Y.-C. Chou, and S.-M. Shen, "High-efficiency, veryhigh color rendering white organic light-emitting diode with a high triplet interlayer," Journal of Materials Chemistry, vol. 21, no. 46, pp. 18523-18526, 2011.

[21] S. Han, C. J. Huang, and Z. H. Lu, "Organic light-emitting devices with silicon anodes," Journal of Applied Physics, vol. 97, no. 9, pp. 086107-086110, 2009.

[22] H. Peng, X. Zhu, J. Sun et al., "Efficient organic light-emitting diode using semitransparent silver as anode," Applied Physics Letters, vol. 87, no. 17, pp. 173505-173507, 2005.

[23] Q. Zhuang, Z. Ding, G. You, Li Kan, H. Zhen, and Q. Ling, "Solution-processed, top-emitting, microcavity polymer light-emitting diodes for the pure red, green, blue and near white emission," Nanotechnology, vol. 31, no. 8, pp. 1-7, 2019. 